OPEN

SUBJECT AREAS:

CELL DEATH IN THE

NERVOUS SYSTEM

CELL DEATH

Received

15 October 2014

Accepted

18 December 2014

Published

2 February 2015

Correspondence and requests for materials should be addressed to W.X. (wlxia@sjtu.edu. cn) or W.-Q.G. (weiqgao@yahoo. com)

* These authors contributed equally to this work.

\section{Adjudin protects rodent cochlear hair cells against gentamicin ototoxicity via the SIRT3-ROS pathway}

\author{
Yizhou Quan ${ }^{1,2 *}$, Li Xia ${ }^{3 *}$, Jiaxiang Shao ${ }^{1,2}$, Shankai Yin ${ }^{3}$, C. Yan Cheng ${ }^{4}$, Weiliang Xia ${ }^{1,2}$ \\ \& Wei-Qiang Gao ${ }^{1,2}$
}

\begin{abstract}
'State Key Laboratory of Oncogenes and Related Genes, Renii-Med X Stem Cell Research Center, Ren Ji Hospital, School of Medicine, Shanghai Jiao Tong University, Shanghai, China, ${ }^{2}$ School of Biomedical Engineering \& Med-X Research Institute, Shanghai Jiao Tong University, Shanghai, China, ${ }^{3}$ Department of Otolaryngology, Affiliated Sixth People's Hospital of Shanghai Jiao Tong University, Otolaryngology Institute of Shanghai Jiao Tong University, Shanghai, China, ${ }^{4}$ The Mary M. Wohlford Laboratory for Male Contraceptive Research, Center for Biomedical Research, Population Council, New York, New York, USA.
\end{abstract}

Hearing loss resulting from hair cell degeneration is a common disease that affects millions of people worldwide. Strategies to overcome the apparent irreversible hair cell loss in mammals become paramount for hearing protection. Here we reported that, by using a well-established gentamicin-induced hair cell loss model in vitro, adjudin, a multi-functional small molecule drug, protected cochlear hair cells from gentamicin damage. Immunohistochemistry, Western blotting and quantitative RT-PCR analyses revealed that adjudin exerted its otoprotective effects by up-regulating the level of Sirt3, a member of Sirtuin family protein located in mitochondria, which regulates reactive oxygen species (ROS) production in cochlear cells and inhibits the production of ROS and apoptotic cells induced by gentamicin. Sirt3 silencing experiments confirmed that Sirt3-ROS signaling axis mediated hair cell protection against gentamicin by adjudin, at least in part. Furthermore, adjudin's otoprotection effects were also observed in an in vivo gentamicin-injured animal model. Taken together, these findings identify adjudin as a novel otoprotective small molecule via elevating Sirt3 levels and Sirt3 may be of therapeutic value in hair cell protection from ototoxic insults.

A uditory hair cells are located in the cochlea and convert sound signals into electrical impulses ${ }^{1}$. Degeneration of hair cells due to aging, acoustic trauma and aminoglycoside antibiotics is a major cause of hearing loss - a disease that affects hundreds of millions of people worldwide ${ }^{2}$. In mammals, hair cell loss is regarded irreversible ${ }^{3}$, and therefore hair cell regeneration by altering expression of specific genes that are responsible for the differentiation of hair cells ${ }^{3,4}$ or stem cell therapy ${ }^{5}$ is likely the solution for hearing function restoration. Strategies for hair cell protection or methods that delay the degeneration process are also actively pursued ${ }^{6}$. By using aminoglycoside antibiotics such as gentamicin as the ototoxic agent ${ }^{7}$, various molecules are found to be hair cell protective either in vitro or in vivo via different mechanisms/pathways. These include Concanavalin A that blocks gentamicin uptake into hair cells ${ }^{7}$, XIAP that inhibits cellular apoptosis ${ }^{8}$, minocycline that attenuates the activation of Caspase $3^{9}$, HGCYT, a newly designed herbal drug that suppresses the activation of Caspase $9^{10}$, and molecules that block c-Jun N-terminal kinase pathway including CEP-1347 ${ }^{11}$, estradiol ${ }^{12}$ and D-JNKI- $1^{13}$. Other molecular targets are also identified, such as HSP70 targeted by GGA ${ }^{14}$ or Geldanamycin ${ }^{15}$, Egb $761^{16}$ and Heme oxygenase- $1^{17}$ that could reduce reactive oxygen species in cochlear hair cells. However, many of these compounds are toxic, thus limiting their usability in vivo. Finding novel molecules that are of little toxicity are clinically desired.

Adjudin, or AF-2364 [1-(2,4-dichlorobenzyl)-1H-indazole-3-carbo-hydrazide], is an analog of lonidamine ${ }^{18}$. Due to its low toxicity and the ability to exfoliate germ cells from the seminiferous epithelium, adjudin is thought to be a potential male contraceptive ${ }^{19}$. We have recently reported that adjudin suppresses NF- $\mathrm{\kappa B}$ activation in microglial cells and mitigates neuroinflammation, thus confers neuroprotection ${ }^{20}$. We hypothesize that adjudin might also have a protective effect on hair cells against gentamicin ototoxicity.

We report here for the first time that adjudin protected cochlear hair cells against gentamicin ototoxicity in vitro and in vivo. We performed immunohistochemistry, gain or loss of function, and rescue experiments to provide evidence that adjudin functions through up-regulation of Sirt3 and suppression of the production of 
ROS. The adjudin-Sirt3-ROS axis underlies the protective mechanism that maintains hair cell integrity and functionality following gentamicin exposure, and possibly other assaults.

\section{Methods}

Ethics statement. All studies were approved by the Institutional Animal Care and Use Committee, Shanghai Jiao Tong University (approval number: BME-2014022), and all animals were treated in accordance with the institution guidelines.

Animals. Cochlear explant cultures were prepared from P4 Sprague-Dawley rats (purchased from SLAC Laboratory, Shanghai Institutes for Biological Science). In vivo functional experiments were carried out in C57BL/6J mice (purchased from Experimental Animal Service of Shanghai Jiao Tong University School of Medicine). In total 96 mice irrespective of gender were selected at 5-week age with body weights around $25 \mathrm{~g}$. All animal procedures were carried out according to the guidelines of Institutional Animal Care and Use Committee, Shanghai Jiao Tong University.

Organotypic cultures of cochlear explants and cochlear cells. Organotypic cultures of cochlear explants and cochlear cells were performed essentially as previously described $^{4,7}$. In brief, before dissecting, collagen gels were coated on the bottom of 24 well plate $(10 \mu \mathrm{L}$ each well) to make substrate-coated wells. Collagen gels (rat tail collagen, type I; $4.08 \mathrm{mg} / \mathrm{mL}$ formulated in $0.02 \mathrm{~N}$ acetic acid) were used as a mixture with DMEM/F12 medium and $2 \%$ sodium carbonate in a ratio of $9: 1: 1$. The coated plate was left in cell incubators at $37^{\circ} \mathrm{C}$ for several minutes till the fluid gels becoming solidified. Cochlear ducts were dissected free of stria vascularis and spiral ganglia. After the tissues were dissected, $500 \mu \mathrm{L}$ of medium [DMEM/F12 plus $10 \%$ FBS; $2 \mathrm{mM}$ glutamine; $25 \mathrm{mM}$ HEPES and $30 \mathrm{U} / \mathrm{mL}$ penicillin] was added to each well in the plate. Then, the middle turn cochlear explants were put in the wells in touch with the collagen gels.

To find out whether adjudin could protect hair cells from gentamicin-induced ototoxicity, the cultures were divided into four groups: Group 1, the cultures were maintained in the normal DMEM/F12 medium for 2 days; Group 2, the cultures were maintained in normal medium for 1 day and then they were challenged with $0.05 \mathrm{mM}$ gentamicin (Gibco) for another day; Group 3, the cultures were pre-treated with adjudin for 1 day and were challenged with $0.05 \mathrm{mM}$ gentamicin in the presence of adjudin for another day; Group 4, cultures were maintained in the medium with adjudin for 2 days. The optimal adjudin concentration was determined based on toxicity and hair cell protective efficiency. Thereafter, the cochlear explants were fixed with $4 \%$ paraformaldehyde for $30 \mathrm{~min}$ and washed in phosphate-buffered saline (PBS), followed by immunofluorescence staining.

For culturing cochlear cells, isolated cochlear tissues were subjected to enzymatic digestion with $0.125 \%$ collagenase for $30 \mathrm{~min}$ at $37^{\circ} \mathrm{C}$, followed by a $5 \mathrm{~min}$ treatment with $0.125 \%$ trypsin. After adding one volume of DMEM/F12 medium with $10 \%$ FBS, the suspension was passed through a $50 \mu \mathrm{m}$ cell strainer (BD Falcon) and the resulting single cells received the same treatment with the cochlear tissues as described above.

Immunofluorescence microscopy. To examine the staining pattern of F-actin in stereociliary arrays of hair cells, the fixed tissues were incubated with $0.5 \mu \mathrm{g} / \mathrm{mL}$ phalloidin-FITC conjugates (Sigma) in PBS for $40 \mathrm{~min}$ at room temperature. To examine the hair cells, the fixed preparations were first permeablized in $1 \%$ Triton X100 for $1 \mathrm{~h}$, blocked with $10 \%$ normal goat serum at room temperature for $30 \mathrm{~min}$, and then incubated with a myosin-VIIa antibody (1:50; Santa Cruz Biotechnology) overnight at $4{ }^{\circ} \mathrm{C}$. Texas red-conjugated secondary antibody $(1: 300$; Jackson ImmunoResearch) were then used to reveal the labeling patterns. Negative controls were performed by skipping the primary antibody step. Primary cochlear cell cultures were fixed in $4 \%$ paraformaldehyde, permeablized in $1 \%$ Triton X-100 for $30 \mathrm{~min}$ and blocked with $10 \%$ normal goat serum for $30 \mathrm{~min}$, then incubated with Anti-SIRT3 antibody $\left(1: 100\right.$; Cell Signal Technology) overnight at $4{ }^{\circ} \mathrm{C}$. Texas red-conjugated secondary antibody ( $1: 400$; Jackson ImmunoResearch) were then used to label the primary antibody. In order to locate the mitochondria, Mito-Tracker Red (Invitrogen) was used to co-label the samples. Cells were first incubated with $400 \mathrm{nM}$ Mito-Tracker Red at $37^{\circ} \mathrm{C}$ for an hour and were washed in serum-free DMEM/F12 medium twice, followed by standard immunostaining procedures. The labeled tissue and cells were visualized under a confocal microscope (Leica SP5 II) and images were processed using Adobe Photoshop software.

Western blot and quantitative RT-PCR. To examine the Sirt3 level, cochlear explants were lysed in RIPA buffer (Millipore, Temecula, CA, USA) supplemented with Complete Protease Inhibitor Cocktail, 2 mM PMSF, and $0.1 \%$ SDS. The protein concentration was measured using the BCA assay kit (Thermo Scientific, Rockford, IL, USA). Total protein $(\sim 30 \mu \mathrm{g})$ was separated by $10 \%$ SDS-PAGE and then transferred to $0.45 \mu \mathrm{m}$ Nitrocellulose Membrane (Millipore). The membrane was blocked with TBST containing $5 \%$ non-fat milk, incubated with anti-SIRT3 antibody (1:100; Cell Signal Technology) at $4{ }^{\circ} \mathrm{C}$ overnight and then hybridized with appropriate HRP-conjugated secondary antibody at room temperature for $1 \mathrm{~h}$. Protein signals were visualized using ECL detection system (Thermo Scientific). For qRT-PCR analysis (ABI Prism 7900HT) of Sirt3, total RNA was isolated by the RNeasy Mini Kit (Qiagen). And cDNA was synthesized from total RNA with PrimeScript 1st Strand cDNA Synthesis Kit (Takara). The primers used are $5^{\prime}$ - gcggctctacacacagaacat- $3^{\prime}$ (forward) and $5^{\prime}$ caggtttcacaacgccagta - $3^{\prime}$ as (reverse). $\beta$-tubulin was used as a control.
Measurement of hROS production in cochlear tissues. After the initial culture period, explants were exposed to the same medium with $0.05 \mathrm{mM}$ gentamicin for 30 hours. At the end of the culture period, the explants were washed once and loaded with APF (12.5 $\mu \mathrm{M}$; Cell Technology), Aminophenyl fluorescein, a special probe which selectively detects highly-reactive oxygen species (hROS) that is mostly associated with cellular damages ${ }^{21}$ by incubation for $45 \mathrm{~min}$ at $37^{\circ} \mathrm{C}$ in humidified air with $5 \% \mathrm{CO}_{2}$. When reacted with hROS (.OH, ONOO-, and $\mathrm{OCl}$ ), APF are converted to fluorescein and exhibit strong, dose-dependent fluorescence ${ }^{21,22}$. The explants were washed in PBS twice and then fixed in 4\% PFA for $30 \mathrm{~min}$. After using the Triton X100 for $20 \mathrm{~min}$, the explants were co-labeled with phalloidin (Sigma) at room temperature for 1 hour.

Analysis of ROS production in cochlear cells. DCFH-DA, 2',7'-dichlorofluorescin diacetatea, which can be converted into the highly fluorescent $2^{\prime}, 7^{\prime}$ dichlorofluorescein (DCF), produced bright green fluorescence in the presence of $\mathrm{ROS}^{23}$. Briefly, cells were cultured in a 24-well plate. After receiving the different treatments, cells were washed twice in PBS and incubated with $10 \mu \mathrm{M}$ DCFH-DA at $37^{\circ} \mathrm{C}$ in a humidified atmosphere of $95 \%$ air and $5 \% \mathrm{CO}_{2}$. After $30 \mathrm{~min}$, the extracellular ROS dye was washed away with DMEM/F12 serum-free medium and the 24-well plate was then put in a microplate reader (BioTek) to read the level of fluorescence. The excitation and emission wavelengths used were $482 \mathrm{~nm}$ and $535 \mathrm{~nm}$.

TUNEL analysis. Cochleae isolated from SD rats were subjected to apoptosis assay. DNA fragmentation of the apoptotic cells were detected by the terminal deoxynucleotidyltransferase-mediated dUTP nick end labeling (TUNEL) technique (DeadEnd $^{\mathrm{TM}}$ Fluorometric TUNEL System, Promega). Firstly, tissues were fixed by $4 \%$ paraformaldehyde for $30 \mathrm{~min}$, permeablized with $1 \%$ TritonX-100 for 1 hour. Then the samples were covered with $100 \mu$ of equilibration buffer for $5 \mathrm{~min}$ at room temperature, replaced by $50 \mu \mathrm{l}$ of terminal deoxynucleotidyltransferase reaction mix containing $4 \mu \mathrm{l}$ fluorescein-12-dUTP and $1 \mu \mathrm{l}$ recombinant terminal deoxynucleotidyltransferase enzyme, and incubated for 1 hour in the dark at $37^{\circ} \mathrm{C}$. TRITC-phalloidin (Sigma) was used to co-label the tissues.

Lentiviral infection. Infection of cochleae was carried out in 24-well plate with serum-free DMEM/F12 medium. Explants were infected with lenti-Sirt3(3 $\times 10^{5}$ infectious units[IU] per well) and lenti-sh-Sirt3 $\left(1.5 \times 10^{6} \mathrm{IU}\right.$ per well $)$ at $37^{\circ} \mathrm{C}$ for 3 hours. Then serum-free DMEM/F12 culture medium was replaced with DMEM/ F12 culture medium plus 10\% FBS and samples were continuously cultured for 4 to 6 days. Lentivirus was purchased from 3DBiopharm Company (3dbiopharm.bioon.com.cn).

Animal models and drug administration. In total, $50 \mathrm{C} 57 \mathrm{BL} / 6 \mathrm{~J}$ mice were maintained on a 12/12-hour light-dark cycle during the experiment ${ }^{24}$, and were randomly appointed to five groups: Group $1(\mathrm{n}=21)$ received a single peritoneal injection of adjudin $(75 \mathrm{mg} / \mathrm{kg}) 12$ hour prior to a single injection of gentamicin $(40 \mathrm{mg} / \mathrm{kg})$ in combination with furosemide $(400 \mathrm{mg} / \mathrm{kg})$; Group $2(\mathrm{n}=23)$ received normal saline $(0.1 \mathrm{ml}$ per subject via i.p.) 12 hours prior to a single injection of gentamicin $(40 \mathrm{mg} / \mathrm{kg})$ in combination with furosemide $(400 \mathrm{mg} / \mathrm{kg})$; Group 3 ( $\mathrm{n}=$ 6 ) were normal controls. The animals in each group received hearing function test before treatment and 3 days, 7 days, 14 days post the injection of gentamicin, respectively. After the functional test, some animals were sacrificed and the cochleae were harvested for morphological observation.

Adjudin was dissolved in corn oil as $10 \mathrm{mg} / \mathrm{ml}$ suspension. A single dose of $75 \mathrm{mg}$ / $\mathrm{kg}$ (i.p.) was given to the animals. Gentamicin (G1914; Sigma-Aldrich; St. Louis, MO, USA) was injected via i.H. to each animal, $40 \mathrm{~min}$ later, furosemide $(400 \mathrm{mg} / \mathrm{kg}$ i.p., cat. \#5913020911; Webster Veterinary, Devens, MA, USA) was applied.

Hearing function tests. All animals were subjected to open-field Auditory Brainstem Response (ABR) tests using Tucker-Davis Technologies (TDT) hardware and software (Alachua, FL, USA) before treatment. Following open-field ABR tests were performed 3, 7, 14 days post-treatment. The acoustic stimuli (tone bursts) were synthesized using SigGen software (10 ms duration; $0.5 \mathrm{~ms}$ rise/fall time; Blackman window), amplified via an SA1 audio amplifier, and transduced by a broadband electrostatic speaker (FT28D). The signals were collected by subdermal electrodes and amplified 20 times; band-pass filters were set between $100 \mathrm{~Hz}$ and $3 \mathrm{kHz}$. The signals were input to a real-time processor (RA16BA), processed using BioSig software (TDT), and preamplified with a gain of 20 and averaged for 1000 sweeps in each trial. The threshold was evaluated at $2,4,8,16$, and $32 \mathrm{kHz}$. At each frequency, the stimuli were presented at $90 \mathrm{~dB}$, decreased in $5 \mathrm{~dB}$ steps until the threshold was approached, and then decreased in $5 \mathrm{~dB}$ steps until the ABR wave disappeared. The threshold was defined as the lowest intensity at which a visible and repeatable ABR wave was observed in two averaged runs. The body temperature of the animals was maintained at $38^{\circ} \mathrm{C}$ with a thermostatic heating pad.

As for compound action potential (CAP) tests, the subjects were anesthetized same as the animals for ABR tests. The stereotaxic restraint was utilized to fix the head of the animals. The auditory bulla was exposed, and then the round window niche was revealed through a hole (approximately $5 \mathrm{~mm}$ diameter) on the mastoid. A silver ball electrode was laid on the round window membrane and led to the top of the skull. Two electrodes were placed subdermally over the vertex. Acoustic signals generating $\mathrm{CAP}$ and the corresponding frequencies to be tested were the same as those used for $\mathrm{ABR}$. At each frequency, measurements were performed in declining steps from 
$90 \mathrm{~dB}$ SPL in $5 \mathrm{~dB}$ decrement. The CAP responses were amplified with a gain of 20 and averaged 100 times. The amplitude between the first negative depression and subsequent positive wave was measured to assess the protective effect of adjudin on CAP input/output (I/O) functions in specific groups.

Stretched preparation of the basilar membrane and hair cell counting. After the ABR or CAP test, the animals at the varied time points were euthanized with an overdose of pentobarbital (200 mg/kg, i.p. injection), and their temporal bones were harvested. Each bulla was opened using rongeurs to expose the cochlea. The oval window and round window were then opened. Following the creation of a hole in the cochlear apex, paraformaldehyde in $0.1 \mathrm{M}$ phosphate-buffered saline (PBS) was perfused through the cochlea for at least $4 \mathrm{~h}$. The cochlea was decalcified in $10 \%$ EDTA on a swing bed for 7 days at room temperature. Subsequently, the basilar membrane was dissected under a dissecting microscope, and the stria vascularis and tectorial membrane were removed. To identify F-actin and cell nucleus in the organ of Corti, rhodamine-phalloidin (5 $\mu \mathrm{g} / \mathrm{ml}$; P1951; Sigma-Aldrich; St. Louis, MO, USA) and DAPI ( $1 \mu \mathrm{g} / \mathrm{ml}$; D9564; Sigma-Aldrich; St. Louis, MO, USA) was applied for $20 \mathrm{~min}$ and $10 \mathrm{~min}$, respectively, at room temperature away from light. The specimens were then rinsed three times with $0.1 \mathrm{M}$ PBS ( $\mathrm{pH}$ 7.4). The fluorescence signals of the hair cells were counted under a Nikon light microscope with epifluorescence (Nikon ECLIPSE 80i, Tokyo, Japan), and the images were taken with Zeiss LSM 710 laser-scanning confocal microscope (Carl Zeiss, Jena, Germany).Acquired images were imported into Adobe Photoshop, resized and cropped, if needed.

Statistical methods. The statistical software GraphPad Prism was used in data processing and analyzed the significance. For cell courting, surviving hair cells were counted from three or four randomly selected fields in the middle turn of each culture. The average of the cell counted from the three or four randomly selected fields was taken as one sample. Data were collected from three or more cultures for each of the experimental groups and are presented as mean values \pm S.E.M. Students' test was used to compare two groups ( $\mathrm{p}<0.05$ was considered significant) and ANOVA analysis with Turkey post hoc was used to compare three or more groups ( $\mathrm{p}<0.05$ was considered significant).

\section{Results}

Adjudin Protects Hair Cells against Gentamicin Ototoxicity. To determine whether adjudin has the capacity of protecting inner hair cells against ototoxic damages, we carried out similar experiments as we previously did for verification of an otoprotection effect in postnatal rat cochlear explant cultures ${ }^{7,25}$. Cochlear explant cultures were subjected to gentamicin challenge (at $0.05 \mathrm{mM}$ ) with adjudin pretreatment at increasing concentrations and then examined for stereociliary bundles of hair cells using phalloidin-FITC staining (Fig. 1A\&B). As shown in Fig. 1A \& Fig. 1B, a dose-dependent protection on hair cells by adjudin against gentamicin was observed and there was a significant protective effect by adjudin (at $100 \mu \mathrm{M}$ ) against gentamicin ototoxicity (Fig. 1A:b vs. e). In parallel with phalloidin (green), a well-known hair cell body marker, myosin VIIa (red) was also used to label the hair cells to assist the determination of survived hair cells in the four groups of cochlear explant cultures. Cultured in normal medium (Fig. 2A: a, e, i) or with adjudin (100 $\mu \mathrm{M}$, Fig. 2A: d, h, l) for 2 days, the hair cells appeared healthy. Addition of adjudin itself to the cultures did not induce any apparent morphological or stereociliary bundle pattern changes at $50-100 \mu \mathrm{M}$, although at $100 \mu \mathrm{M}$ very little disorganization of stereocilia start to appear. The majority of hair cells did not survive gentamicin challenge (Fig. 2A: b, f, j), appearing disorganized and fragmented based on Myosin VIIa staining and phalloidin labeling (Fig. 2A: b, f, j). In contrast, in 1 day adjudin-pretreated cultures (Fig. 2A: c, g, k), many of the hair cells survived gentamicin insult. Not only hair cell bodies but also their stereociliary bundles, especially the outer hair cells, exhibited normal morphological features. The number of surviving hair cells was also quantified in the different treatment groups from 4-5 experiments (Fig. 2B), revealing a protective effect by adjudin (Fig. 2A:b, f, j vs. c, g, $\mathrm{k})$. In addition, the number of apoptotic cells in the cochlear was also examined (Fig. 2C). Gentamicin treatment resulted in more TUNEL ${ }^{+}$ apoptotic cells in the Organ of Corti (Fig. 2C: b), while pretreatment with adjudin remarkably reduced cellular apoptosis (Fig. 2C: c). Analyses from 4-5 batches of experiments were summarized in Fig. 2D. Taken together, adjudin pretreatment significantly protected hair cells against gentamicin damage.

Adjudin elevates Sirt3 Expression and Suppresses ROS Level in the Cochlea. We then explored the mechanisms underlying adjudin's protective function. Adjudin is known to be a mitochondria-targeting drug that could activate a number of pathways ${ }^{20}$. As Sirt 3 induction is
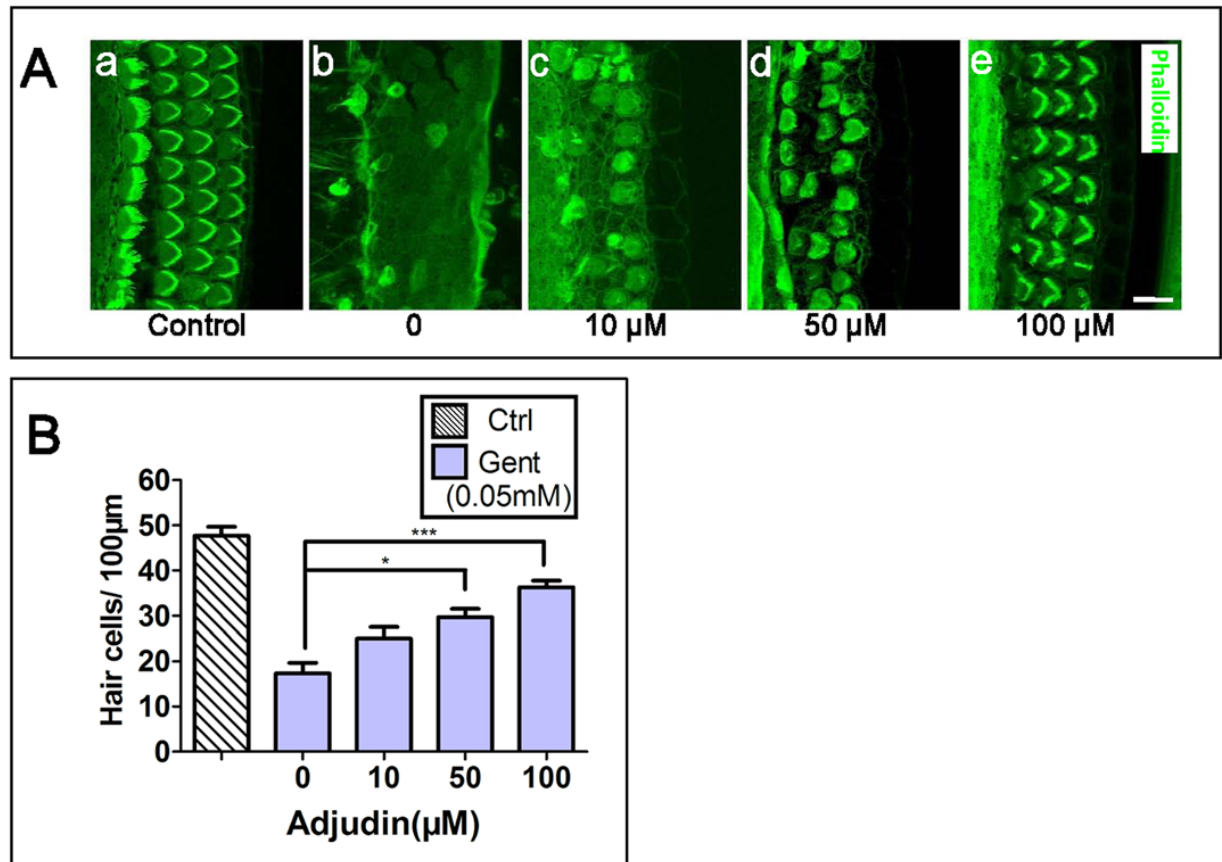

Figure 1 Titration of adjudin concentrations on hair cell protection against gentamicin ototoxicity in cochlear explant cultures maintained for 2 days in vitro (DIV). (A) Gentamicin $(0.05 \mathrm{mM})$ was added in (b,c,d,e) but not in (a). Different concentrations of adjudin were added from $10 \mu \mathrm{M}(\mathrm{c})$, $50 \mu \mathrm{M}$ (d) to $100 \mu \mathrm{M}$ (e). Samples were labeled with phalloidin (green). Bar $=10 \mu \mathrm{m}$. (B) Analysis of hair cell numbers in samples as treated in (A). Adj, adjudin. Data were collected from six or more cultures in each group and are expressed as means \pm SEM. ${ }^{*} \mathrm{p}<0.05$; $^{* * *} \mathrm{p}<0.001$, one-way ANOVA followed by Tukey's multiple comparison test. 

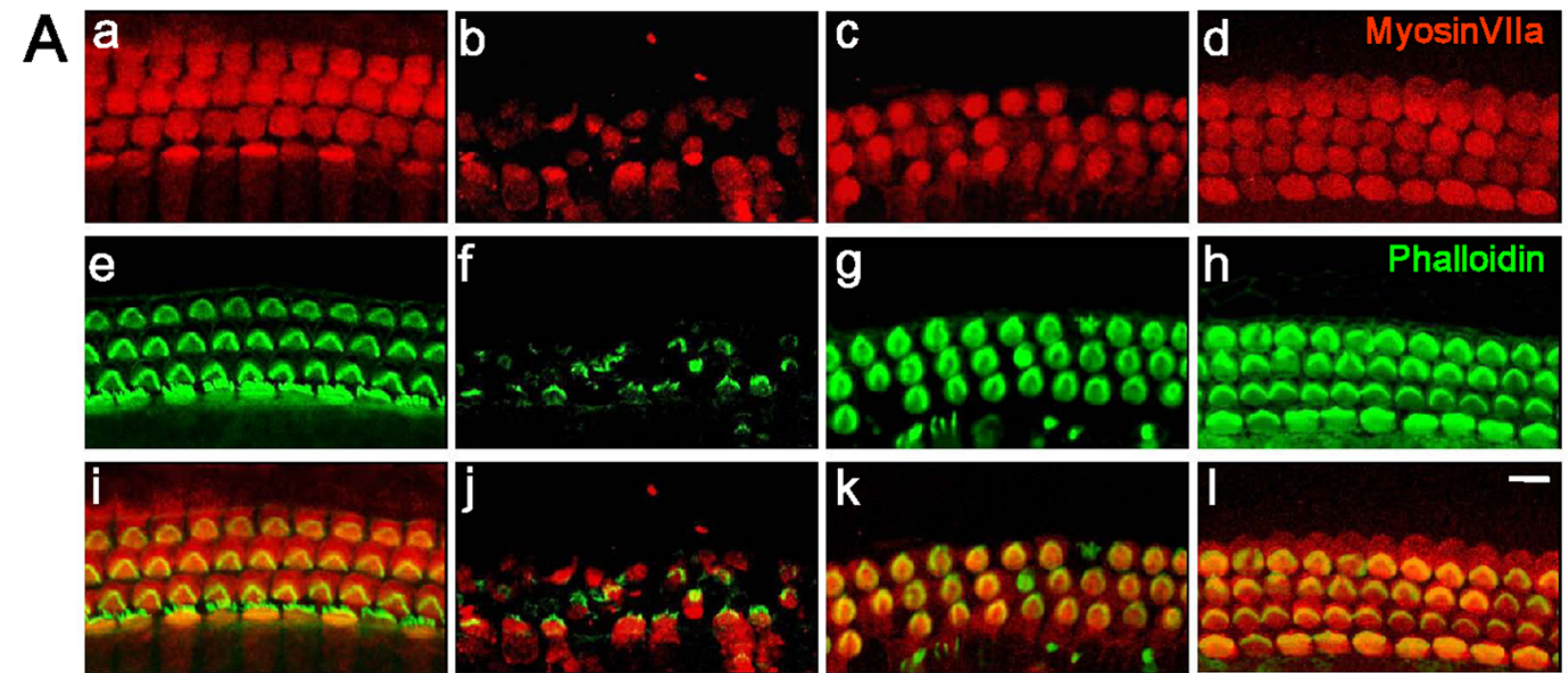

Ctrl

Gent

Adj+Gent
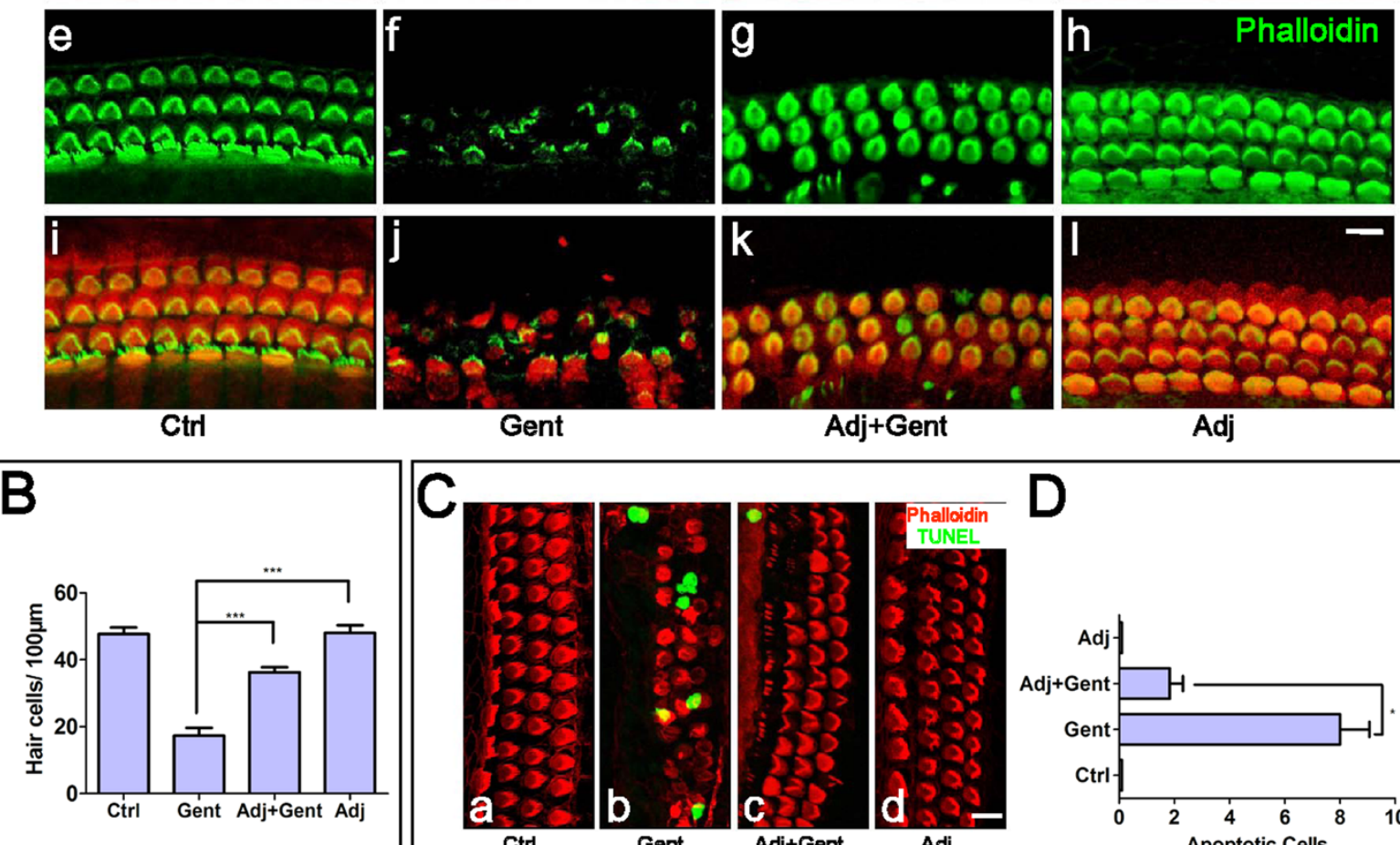

Ctrl

Gent
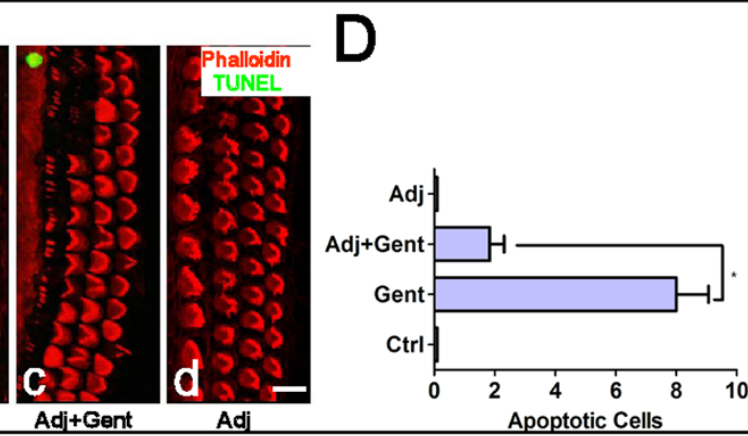

Figure $2 \mid$ Adjudin protects against gentamicin-induced hair cell loss in rat cochleae. (A) Samples from the four treatment groups were labeled with Myosin VIIa (red, a-d) and Phalloidin (green, e-h), with the merged images shown in (i-l). Cochlear explant cultures were divided into four treatment groups: (1) no treatment control (a, e, i); (2) a culture treated with $0.05 \mathrm{mM}$ gentamicin (b, f, j). Note that most of the hair cells were depleted; (3) a culture which was pretreated with $100 \mu \mathrm{M}$ adjudin for one day and then challenged with $0.05 \mathrm{mM}$ gentamicin in the presence of adjudin for another day (c, g, $\mathrm{k}$ ); and (4) a culture which was treated with $100 \mu \mathrm{M}$ adjudin for 2 days $(\mathrm{d}, \mathrm{h}, \mathrm{l})$. Note that adjudin has little cytotoxicity itself. Bar $=10 \mu \mathrm{m}$. (B) Quantitative analysis of the number of hair cells in the different treatment groups as shown in the representative images in (A). (C) Adjudin reduces gentamicin induced-hair cell apoptosis. Cochlear explant cultures were divided into four groups: (a) control; (b) $0.05 \mathrm{mM}$ gentamicin treatment (27 h); (c) Adjudin $(100 \mu \mathrm{M})$ pretreatment for a day followed by $0.05 \mathrm{mM}$ gentamicin challenge in the presence of adjudin for another $27 \mathrm{~h}$; and (d) Adjudin (100 $\mu \mathrm{M})$ treatment only as in (c). Phalloidin (red) labels hair cells whereas the green fluorescence indicates apoptotic hair cells (TUNEL). Bar $=10 \mu \mathrm{m}$.

(D) Quantitative analysis of the number of apoptotic hair cells in the Organ of Corti. Data were collected from five or more cultures in each group and are expressed as means \pm SEM. ${ }^{* * *} \mathrm{p}<0.001,{ }^{*} \mathrm{p}<0.05$, one-way ANOVA followed by Tukey's multiple comparison test.

recently reported to ameliorate hearing loss due to aging in mice through repressing $\operatorname{ROS}^{26}$, we speculate whether Sirt3 could also play a role in hearing dysfunction caused by other factors, such as gentamicin ototoxicity. We then examined whether adjudin could affect Sirt3 expression levels in cochlear cells. Primary cochlear cells were treated with adjudin $(100 \mu \mathrm{M})$, stained with MitoTracker, a mitochondrion marker (Fig. 3A: c, d; red) and anti-Sirt3 antibody (Fig. 3A: e, f; green). Sirt3 was found to co-localize with mitochondria (Fig. 3A: g, h), with the fluorescence signal intensified after adjudin treatment. To corroborate this, cochlear explant cultures were treated with adjudin, and total protein or RNA was extracted, followed by Western blot and quantitative real-time PCR analysis (Fig. 3B). These findings together indicate that adjudin increased both Sirt3 protein levels and mRNA expression in the rat cochlear tissues.

Because Sirt3 is shown to decrease ROS production, we next performed experiments to determine whether ROS production could be reduced by adjudin treatment using 2- [6-( $4^{\prime}$-amino) phenoxy-3Hxanthen-3-on-9-yl]benzoic acid, also known as APF, a special probe which selectively detects highly-reactive oxygen species (hROS, .OH, ONOO-, and -OCl) that are mostly associated with cellular damage $e^{1,17,27}$. First, ROS production from the whole epithelium of the cochlea was examined. Cochleae treated with gentamicin had more hROS positive cells than the three other groups (Fig. 4A: ad). It was noticed that even in the control group (Fig. 4A: a) there were still some ROS positive cells. In contrast, little ROS production was found in cultures treated by adjudin, with or without gentamicin (Fig. 4A: c, d). Quantitative analysis of ROS positive cells in the cochlear epithelium (Fig. 4A: e) confirmed that this adjudin-induced ROS suppression was statistically significant. Generally, when the cultures were treated with adjudin for 2 days, the level of cochlear Sirt3 protein was nearly tripled while hROS production was reduced by more than half in cochlear epithelia compared to tissues without adjudin treatment (Fig. 4A: f). ROS levels in the isolated primary cochlear cells were also measured by DCFH-DA, another common method to measure ROS levels. These experiments confirmed that adjudin could quench ROS production (Fig. 4B). In addition, to determine the ROS production in the Organ of Corti under the different treatment conditions, phalloidin and APF were both applied to reveal the actin bundles of the hair cells and the ROS signals (Fig. 4C). Gentamicin treatment induced more ROS positive 


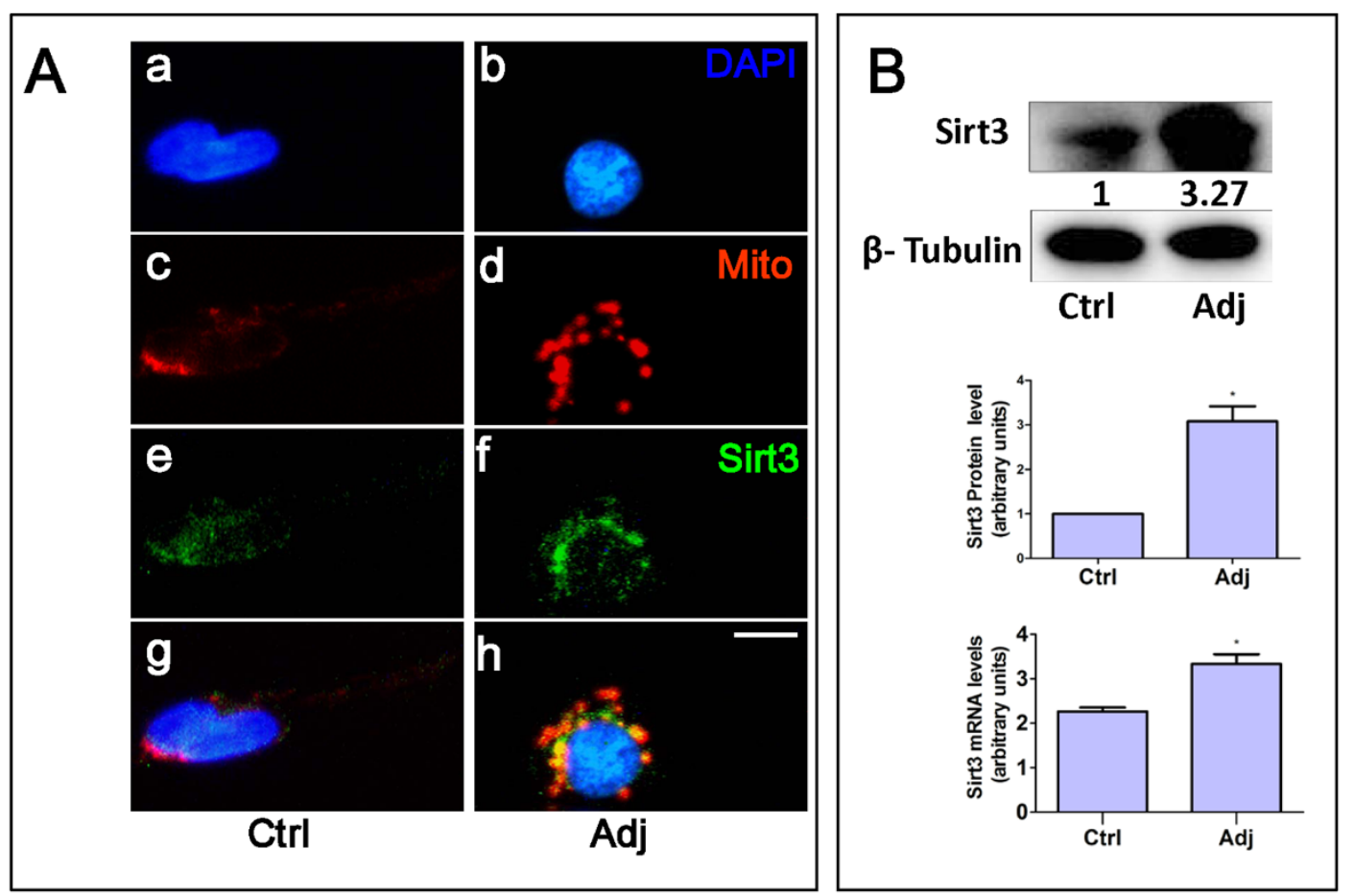

Figure 3 Adjudin stimulates Sirt3 expression in cultured primary cochlear cells and the Organ of Corti. (A) Immunofluorescence analysis in cultured cochlear cells treated with or without adjudin $(100 \mu \mathrm{M})$ : DAPI (nucleus; a, b); Mito-Tracker Red (mitochondria; c, d); Anti-Sirt3 antibody (green; e, f); and their merged images $(\mathrm{g}, \mathrm{h})$. Bar $=10 \mu \mathrm{m}$. (B) Top: Western blot analysis of Sirt3 protein levels from cochlear samples treated with or without adjudin $(100 \mu \mathrm{M})$ and densitometric quantifications of Sirt3 levels are indicated. Below: Q-PCR measurement of Sirt3 expression in cochlear cells after adjudin $(100 \mu \mathrm{M})$ treatment, tubulin was used as a loading control. Data were collected from three or more cultures in each group and are expressed as means \pm SEM, ${ }^{*} \mathrm{p}<0.05$, student's t test.

cells accompanied by the reduction of hair cell numbers (Fig. 4C: b, f, $\mathrm{j}$ ), whereas treatment of adjudin significantly reduced ROS positive cells and conserved more hair cells (Fig. 4C: c, g, k).

Sirt3 Protects Hair Cells from Gentamicin Ototoxicity. To further confirm whether Sirt3 mediated adjudin's protective function on gentamicin ototoxicity, we further designed gain- and loss-offunction assays in cochlear explant cultures. Cochlear explants were infected with lentivirus (lenti-Sirt3) to overexpress Sirt3 (Fig. 5A). Control virus (Vector) was included in parallel. LentiSirt3 was able to induce Sirt3 overexpression in cochlear explants (Fig. 5A: f). Four days after infection, these cultures were subjected to gentamicin challenge for 2 days, followed by hair cell morphological examination using phalloidin-Texas Red. Infection of vector and lenti-Sirt3 apparently had little effect on the hair cell (Fig. 5A: ab). Overexpression of Sirt 3 in cochleae rescued partially gentamicininduced hair cell loss (Fig. 5A: c vs. d). Hair cell numbers in cochlear explants of these treatment groups from 4-5 batches of experiments were summarized (Fig. 5A: e), which showed that an overproduction of Sirt 3 could attenuate gentamicin-induced hair cell loss.

Adjudin Exerts its Otoprotection via the Sirt3-ROS Pathway. To provide additional evidence to support our model that adjudin exerts its otoprotection via the Sirt3-ROS pathway, we then used the lentivirus expressing Sirt3-specific short hairpin RNA (sh-Sirt3) to knock down Sirt3 level in the cochlea (Fig. 5B). This lentivirus could effectively down-regulate Sirt3 expression (Fig. 5B: f). Lentivirus that expresses non-targeting shRNA (NT shRNA) and sh-Sirt3 apparently did not affect hair cell morphology (Fig. 5B: ab). Four days after infection, these cultures were treated with gentamicin in the presence of adjudin for 2 days (Fig. 5B: c-d). When Sirt3 level was down-regulated, however, adjudin failed to protect hair cells against gentamicin damage as effectively (Fig. 5B: c vs. d). This experiment was repeated 4-5 times and the number of hair cells in the different treatment groups was analyzed (Fig. 5B: e). Together these results indicate that Sirt3 mediated, at least in part, the hair cell protective effects by adjudin. It is noted that knockdown of Sirt3 could only partially reverse adjudin's function (Fig. 5A: e vs. Fig. 5B: e), suggesting that there might be other signals involved in this process. Another possibility is that adjudin treatment affects all cells whereas lentivirus infects a subset of cells. Whole mount cochlear tissues infected with lentivirus and sh-virus were shown in suppl. Fig. S1.

Adjudin Protects Gentamicin Ototoxicity in vivo. To further verify if adjudin could confer hearing protection against gentamicin in vivo, we performed various histological and functional assays in a gentamicin injury mouse model (see Methods). The time line of the experiments for the protective effects by adjudin against ototoxicity of gentamicin in vivo is shown in Fig. 6A. In the group of mice that received adjudin and gentamicin $(40 \mathrm{mg} / \mathrm{kg})$ application, an evident protective effect of adjudin was observed at 14 days post the injection. The amount of OHC loss was approximately 30\% compared with that of about $60 \%$ in the group without adjudin injection (Fig. 7A: a and Fig. 7B: b vs. d). However, at 7 days post adjudin administration (Fig. 7B: a vs. c), no significant protection was seen (Suppl. Fig. S2).

To determine if there is a protection of hearing function by adjudin against gentamicin, auditory brainstem response (ABR) and compound action potential (CAP) were recorded for the groups of normal control group and the groups receiving gentamicin with and without adjudin administration, at various observing time points post drug applications $(n=6$ ears for each group, one from each animal). The animals in the normal control group showed no significant differences of $\mathrm{ABR}$ threshold shifts for each frequency between the starting and the terminal points of the experimental 


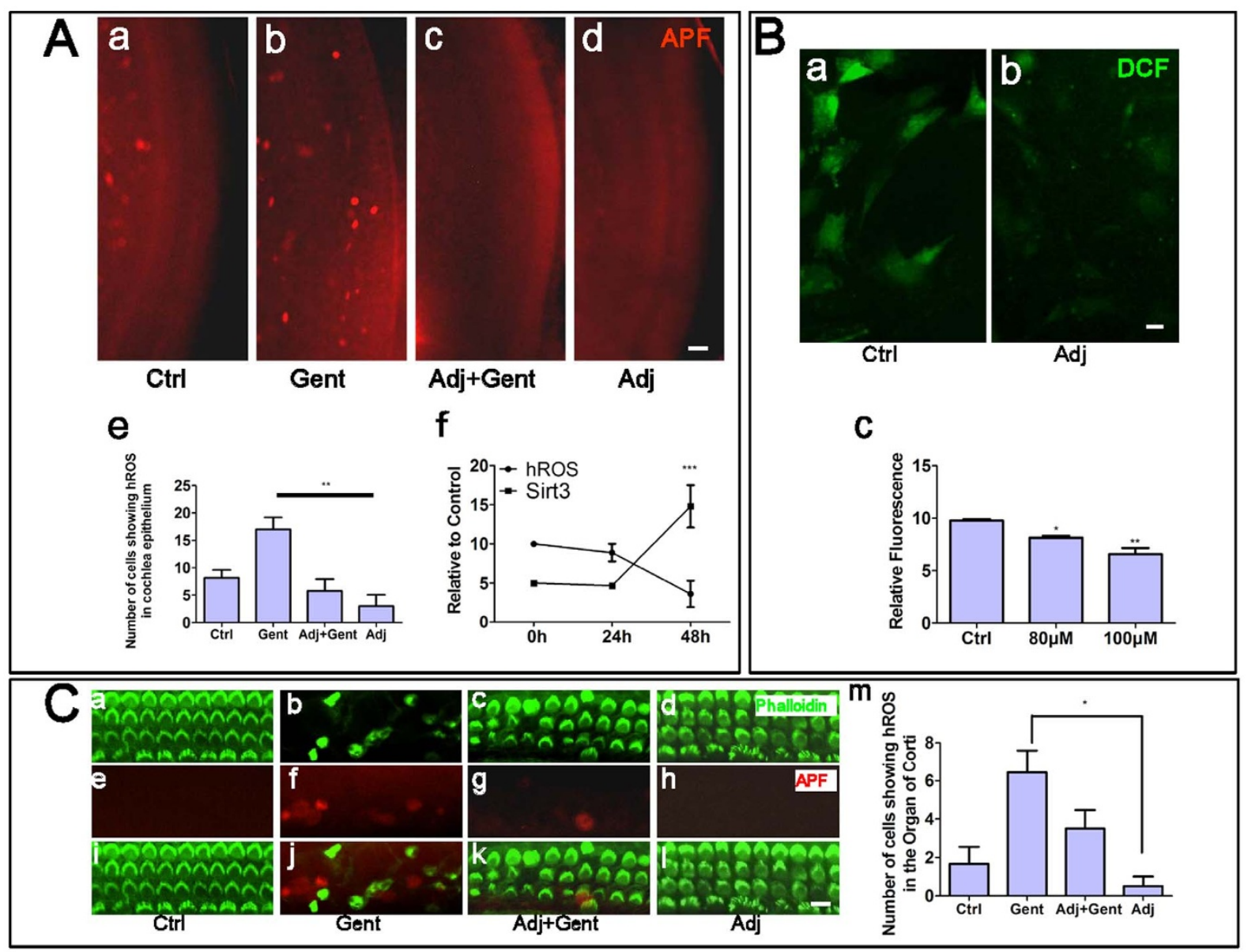

Figure $4 \mid$ Adjudin suppresses ROS production in the epithelium of cochlea and in primary cochlear cells. (A) Cochlear explant cultures were divided into four groups: (a) control; (b) $0.05 \mathrm{mM}$ gentamicin treatment $(30 \mathrm{~h})$; (c) Adjudin $(100 \mu \mathrm{M})$ pretreatment for a day followed by $0.05 \mathrm{mM}$ gentamicin challenge in the presence of adjudin for another $30 \mathrm{~h}$; and (d) Adjudin $(100 \mu \mathrm{M})$ treatment only as in (c). The fluorescence (spotted signals) labels ROS positive cells (APF, red). (e) Quantitative analysis of the number of ROS positive cells in cochlear epithelium cultures from the four treatment groups. ** $\mathrm{p}$ $<0.01$, one-way ANOVA followed by Tukey's multiple comparison test. (f) The relationship between the protein level of SIRT3 and hROS production in cochlear epithelia samples when adjudin was added for 2 days. ${ }^{* *} \mathrm{p}<0.001$, two-way ANOVA of the parameters between various regimens. Bar $=$ $25 \mu \mathrm{m}$. (B) Primary cochlear cells were treated with or without adjudin $(100 \mu \mathrm{M})$ and labeled for ROS level with DCF (a, b), and the ROS production in cochlear cells were quantified in (c). Data were collected from five or more cultures in each group and are expressed as means \pm SEM. $* \mathrm{p}<0.05, * * \mathrm{p}<$ 0.01, one-way ANOVA followed by Tukey's multiple comparison test. Bar $=5 \mu \mathrm{m}$. Adjudin suppresses hROS production in the Organ of Corti.

(C) Cochlear explant cultures were divided into four groups: control (a,e,i); $0.05 \mathrm{mM}$ gentamicin treatment for $30 \mathrm{~h}$ (b,f,j); (c) Adjudin (100 $\mu \mathrm{M})$ pretreatment for a day followed by $0.05 \mathrm{mM}$ gentamicin challenge in the presence of adjudin for another $30 \mathrm{~h}(\mathrm{c}, \mathrm{g}, \mathrm{k})$; and adjudin $(100 \mu \mathrm{M})$ treatment only (d,h,l). Phalloidin (green) (a-d); APF (red) stained the cells with high ROS production (e-h) and merged images $(\mathrm{i}-\mathrm{l})$. Bar $=10 \mu \mathrm{m}$.

(m) Quantitative analysis of the number of ROS positive cells in the Organ of Corti. Data were collected from five or more cultures in each group and are expressed as means \pm SEM. ${ }^{*} \mathrm{p}<0.05$, one-way ANOVA followed by Tukey's multiple comparison test.

time course. The ABR threshold shifts were elevated with gentamicin and especially a big shift was observed at $32 \mathrm{kHz}$ (Fig. 6B), and the hearing function became significantly deteriorated from 7-day to 14 day post-gentamicin treatment. A protective effect was mainly seen for $32 \mathrm{kHz}$ at both 7 days and 14 days post co-injection of gentamicin and adjudin (Fig. 6B: a \& Fig. 6B: b). At 7 days post treatment, the adjudin group showed a less threshold elevation at $32 \mathrm{kHz}(16 \pm$ $1 \mathrm{~dB})$, compared with those receiving normal saline plus gentamicin $(21 \pm 1 \mathrm{~dB}$ ) (Fig. 6B: a). A better protective effect was observed 14 days post injection (Fig. 6B: b). The adjudin group displayed a less threshold shift $(19 \pm 1 \mathrm{~dB})$ at $32 \mathrm{kHz}$ than the control group $(30 \pm$ $1 \mathrm{~dB})$.

The CAP amplitudes recorded at 14-day post-treatment were significantly decreased at $32 \mathrm{kHz}$. Particularly, the amplitudes corresponding to each sound level in the group of adjudin + gentamicin at 14-day post treatment were higher than those in the group of gentamicin alone (Fig. 7A: b). The threshold shifts for $32 \mathrm{kHz}$ measured via CAP between the groups of $40 \mathrm{mg} / \mathrm{kg}$ gentamicin injection with and without adjudin application at 14-day post-treatment were approximately $10-15 \mathrm{~dB}$, in accordance with those via $\mathrm{ABR}$ tests $(\sim 10 \mathrm{~dB}$, Fig. 6B: b).

\section{Discussion}

Hair cell loss and its protection is a central problem for hearing studies. In this work, we report for the first time that adjudin, a novel small molecule drug, protects hair cells against gentamicin ototoxicity by activating Sirt 3 and reducing ROS both in vitro and in vivo. Overexpression of Sirt3 alone partially rescues gentamicin-induced hair cell loss, while Sirt3 inhibition attenuates protection from adjudin treatment. Our study extends the functional role of Sirt3 in hearing protection from ageing to aminoglycoside antibioticsinduced damage. More importantly, by using a generally safe small molecule drug adjudin to activate Sirt3, we provide a new strategy for hair cell protection. 


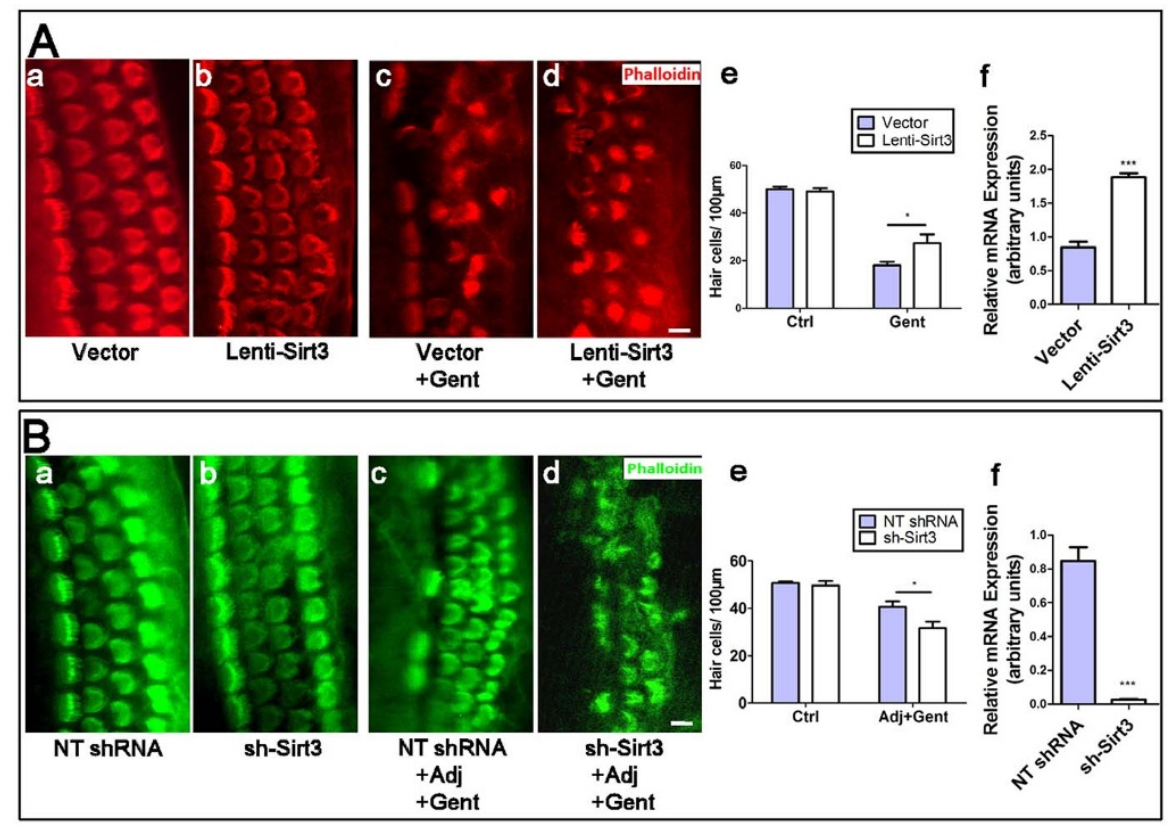

Figure $5 \mid$ Sirt3 mediated, at least partially, hair cell protection by adjudin. (A) Effect of Sirt3 overexpression in cochlear explant culture on gentamicininduced hair cell damage. Cultures were infected with control lentivirus-Vector (a, c) or lenti-Sirt3 (b, d), and labeled with TRITC-phalloidin. Additional gentamicin treatment was indicated (c, d). Quantitative analysis of hair cell numbers in cochlear explant cultures (a-d) was summarized in (e). Q-PCR measurement of relative Sirt3 mRNA levels in Vector or lenti-Sirt3 treated cochlear explant was shown in (f). (B) Knockdown of Sirt3 expression in cochlear explant culture attenuated hair cell protection by adjudin. Cultures were infected with NT shRNA (a, c) or sh-Sirt3 (b, d), and labeled with FITC-phalloidin. Additional treatment with gentamicin + adjudin was indicated $(c, d)$. Quantitative analysis of hair cell numbers in cochlear explant cultures (a-d) was summarized in (e). Q-PCR measurement of relative Sirt3 mRNA levels in NT-shRNA or sh-Sirt3 treated cochlear explant was shown in (f). NT shRNA, Nontargeting control shRNA. Data were collected from five or more cultures in each group and are expressed as means \pm SEM. (A:e, B:e) *p $<0.05 ; * * p<0.01$, two-way ANOVA of the parameters between various regimens, followed by post-hoc tests; (A:f, B:f) $* * * \mathrm{p}<0.001$, student's $\mathrm{t}$ test. Bar $=10 \mu \mathrm{m}$.

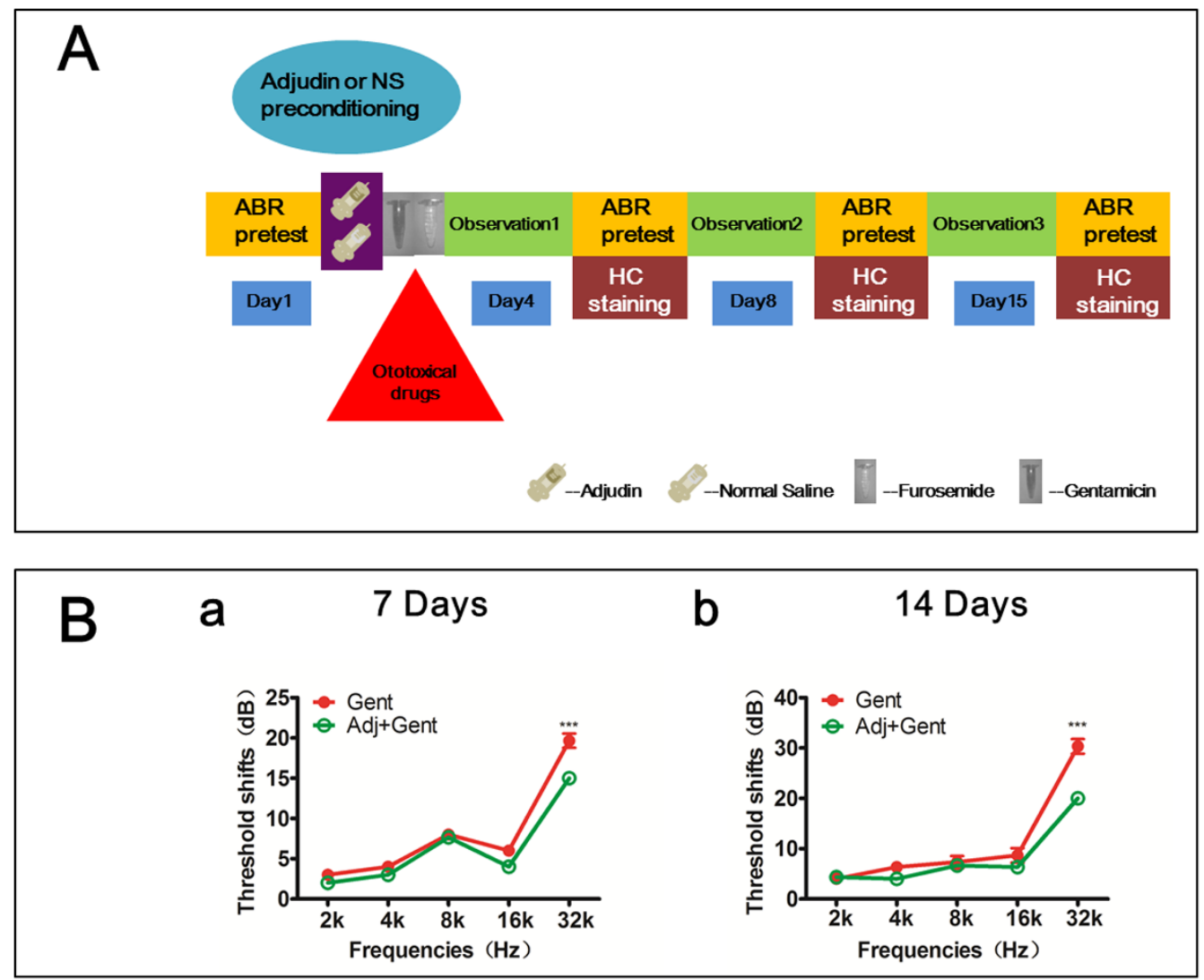

Figure 6 In vivo study of the protective effect of adjudin on cochlear hair cells. (A) The time line of adjudin protecting against ototoxicity induced via gentamicin in vivo. (B) ABR threshold shifts across frequencies 7 days (a) and 14 days (b) after the gentamicin (40 mg/kg) and adjudin (75 mg/kg) administrations $(* * * \mathrm{p}<0.001)$. All statistical analyses $f$ were conducted via two-way ANOVA of the parameters between various regimens, followed by posthoc tests (Tukey Test). 

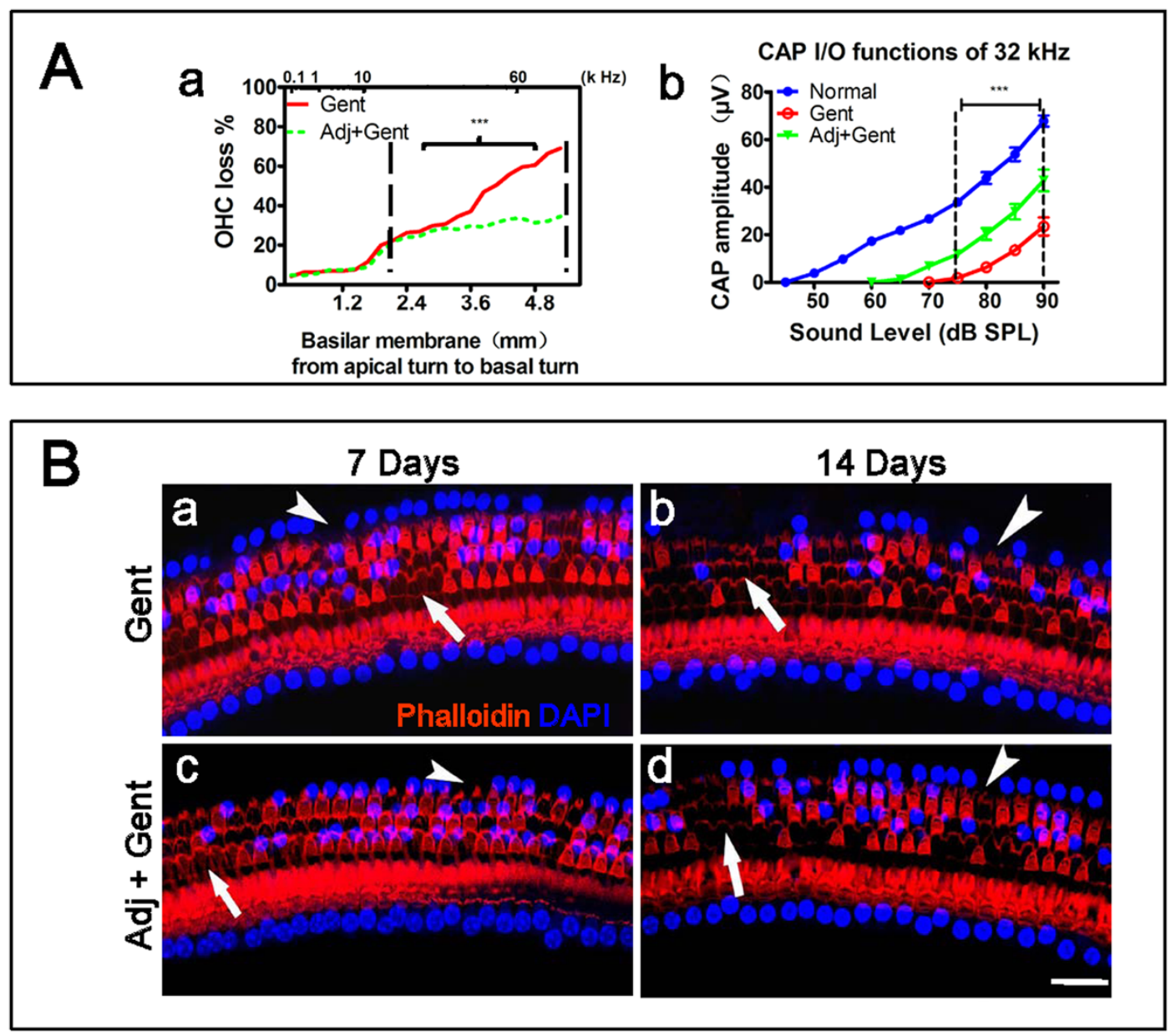

Figure 7 In vivo study of the protective effect of adjudin on cochlear hair cells. (A) Quantification of outer hair cell loss, analyses of CAP input-output functions against baseline, at 14 days post gentamicin treatment $(40 \mathrm{mg} / \mathrm{kg})$; and 14 days post gentamicin $(40 \mathrm{mg} / \mathrm{kg}) \mathrm{plus}$ adjudin $(75 \mathrm{mg} / \mathrm{kg})$ treatment. Cochleogram for OHC loss (A a): Significant difference of OHC loss was found in the basal turn (from $2 \mathrm{~mm}$ to $5.3 \mathrm{~mm}$, beginning at apex between the groups of gentamicin treatment with and without adjudin $(75 \mathrm{mg} / \mathrm{kg})(* * * \mathrm{p}<0.001)$. (A b): CAP input-output functions at $32 \mathrm{kHz}$ at 14 days $(* * * \mathrm{p}<0.001)$. (B) Representative images of surface preparations from different groups: 7 and 14 days post gentamicin treatment $(40 \mathrm{mg} / \mathrm{kg})$ (a, b); and 14 days post gentamicin $(40 \mathrm{mg} / \mathrm{kg}$ ) plus adjudin $(75 \mathrm{mg} / \mathrm{kg}$ ) treatment (c, d). Hair cells were labeled with phalloidin (red) and DAPI (blue). The arrowhead points to the loss of nucleus (blue) whilst the arrow indicates the loss of stereocilia (red). Bar $=50 \mu \mathrm{m}$. All statistical analyses in vivo were conducted via two-way ANOVA of the parameters between various regimens, followed by post-hoc tests (Tukey Test).

Our findings that Sirt3 mediate the otoprotective effects by adjudin against gentamicin are in agreement with the roles of Sirtuins in ageing and metabolism ${ }^{28,29}$. In particular, Sirt3 has been reported to stimulate an enzyme called isocitrate dehydrogenase 2 (Idh2) which converts $\mathrm{NADP}^{+}$to NADPH in mitochondria, leading to increased ratio of reduced glutathione/oxidized glutathione and decreases the production of ROS, thus prevents the age-related hearing loss under the condition of caloric restriction ${ }^{26}$. Along this line, it is important to find small molecules that could activate Sirtuins (Sirt3) and thus down-regulating ROS production. In the present experiments, we show clearly that adjudin can up-regulate Sirt3 expression in cochlear cells and explant cultures, and in turn protects hair cells from gentamicin ototoxicity. In our study, blocking of Sirt3 by lentivirus could only partly abolish the hair cell protection by adjudin suggesting that adjudin possibly acts on other pathways. One possibility is that adjudin might also suppress NF- $\kappa \mathrm{B}$ activation ${ }^{20}$, which requires future studies to verify. Alternatively, such partial blocking effects might be due to the possibility that adjudin treatment affects all cells whereas lentivirus infects only a subset of cells.

While our study indicates that adjudin, a small molecule compound and relatively safe in vivo, may emerge as a promising drug for hearing protection, it would be interesting to evaluate its other potential effects in the inner ear. Adjudin not only demonstrates a potent protective effect on hair cells in vitro as evidenced by immunocytochemical staining with hair cell markers and TUNEL staining, but also shows an in vivo effect in a gentamicin-injury induced hearing loss mouse model as revealed by histology and electrophysiology of hearing threshold shifts and CAPs. On the other hand, hair cell regeneration is believed to be a better way to recover hearing impairment due to hair cell loss, the potential effect of Sirt3 activation, or adjudin treatment on hair cell regeneration, can be investigated in future studies. We are currently constructing a loxP-STOP-loxP-Sirt3 mouse model, which will help the study to determine the possible role of Sirt3 during regeneration of new hair cells or self-repair of partially damaged hair cells ${ }^{7}$.

One limitation of the current study is the cell specificity. We dissected whole mount cochlear tissues from rodent inner ears, and prepared organ cultures which were treated with drugs or infected with lentivirus. Based on the experimental procedures, it is likely that the effect of adjudin is not specific to hair cells alone. First, gentamicin damages and produces ROS in whole mount cochlear cultures that include hair cells and surrounding epithelial cells and adjudin blocks ROS production in both hair cells and other adjacent epithelial cells in the Organ of Corti (both IHC and OHC) as shown by 
immunofluorescence microscopy (Fig. 4A, whole mount cochleae, and Fig. 4C, Organ of Corti). Second, lentivirus infection up-regulated Sirt3 level in these two types of cells in whole mount cochlear tissues as well (Fig 5Af \& Fig S1). Third, up-regulation of Sirt3 expression was induced by adjudin in whole mount cochlear tissues as shown by Western blot analysis (Fig. 3B). Together these data suggest that adjudin might elicit both hair cell autonomous and non-autonomous effects. In other words, the protective effects by adjudin on hair cells could be either directly on hair cells or indirectly mediated by surrounding epithelial cells. In future studies, hair cellspecific transgenic mice using Cre-LoxP system could be used to better clarify this issue.

It is noted that the differences in thresholds in the in vivo experiments were primarily observed at the $32 \mathrm{kHz}$ frequency but appeared undetectable at lower frequency between groups treated with or without adjudin. It is also notable that at 7 days post adjudin administration, no significant protection was seen. We offer the following explanations. Single shot of gentamicin at low doses coupled with furosemide could only damage $\mathrm{OHC}$ in the basal turn and higherfrequency hearing function, because stria vascularis could only open for a short window phase. As described by $\mathrm{Li}$ and coworkers ${ }^{30}$, the $\mathrm{OHC}$ losses after varied doses of an ototoxic drug were huge. Hence, in our studies, $40 \mathrm{mg} / \mathrm{kg}$ gentamicin caused relatively less OHC loss. However, $60 \mathrm{mg} / \mathrm{kg}$ gentamicin combined with furosemide resulted in a more severe damage in $\mathrm{OHC}$, presenting $60 \%$ losses in average with approximately $50 \%$ in the apex and $70 \%$ in the base (unpublished observations).We admitted the limitation of in vivo use of adjudin in the current study. It could only protect hair cells in basal turn against ototoxicity at a certain time points, and the result of hearing function tests roughly corresponded to morphological measurement. One possible explanation for such limited effects by adjudin is presumably related to the poor blood supply in apex where adjudin failed to reach a sufficient working concentration. It is also generally believed that hair cells are more resistant to damage in the apex than those in the base $\mathrm{e}^{31,32}$. In addition, previous studies have shown that the damage of hair cells gets worse at 2-4 wks post gentamicin treatment ${ }^{33}$. The protective effect was not evident on 7 th day presumably due to the fact that hair cell loss was not significant at this time point post gentamicin treatment. However, at 14th day post gentamicin administration, hair cell loss was more evident and protective effects by adjudin became significant based on the different numbers of survived hair cells in the control and adjudintreated mice.

In summary, we have shown that adjudin, mediated by the Sirt3ROS axis, protects cochlear hair cells from gentamicin damage. This molecule may be used as a biochemical tool for the study of mammalian hair cell protection, and has the potential to be used as a treatment option to protect hearing loss.

1. Hudspeth, A. J. How hearing happens. Neuron 19, 947-950 (1997).

2. Nadol, J. B. Jr. Hearing loss. N Engl J Med 329, 1092-1102, doi:10.1056/ NEJM199310073291507 (1993).

3. Mizutari, K. et al. Notch inhibition induces cochlear hair cell regeneration and recovery of hearing after acoustic trauma. Neuron 77, 58-69, doi:10.1016/ j.neuron.2012.10.032 (2013).

4. Zheng, J. L. \& Gao, W. Q. Overexpression of Math1 induces robust production of extra hair cells in postnatal rat inner ears. Nat Neurosci 3, 580-586, doi:10.1038/ 75753 (2000).

5. Okano, T. \& Kelley, M. W. Stem cell therapy for the inner ear: recent advances and future directions. Trends Amplif 16, 4-18, doi:10.1177/1084713812440336 (2012).

6. Rubel, E. W., Furrer, S. A. \& Stone, J. S. A brief history of hair cell regeneration research and speculations on the future. Hear Res 297, 42-51, doi:10.1016/ j.heares.2012.12.014 (2013).

7. Zheng, J. L. \& Gao, W. Q. Concanavalin A protects hair cells against gentamicin ototoxicity in rat cochlear explant cultures. J Neurobiol 39, 29-40 (1999).

8. Tabuchi, K., Pak, K., Chavez, E. \& Ryan, A. F. Role of inhibitor of apoptosis protein in gentamicin-induced cochlear hair cell damage. Neuroscience 149, 213-222, doi:10.1016/j.neuroscience.2007.06.061 (2007).
9. Wei, X. et al. Minocycline prevents gentamicin-induced ototoxicity by inhibiting p38 MAP kinase phosphorylation and caspase 3 activation. Neuroscience 131, 513-521, doi:10.1016/j.neuroscience.2004.11.014 (2005).

10. Kim, S. J. et al. Hwanggunchungyitang prevents cadmium-induced ototoxicity through suppression of the activation of caspase- 9 and extracellular signal-related kinase in auditory HEI-OC1 cells. Biol Pharm Bull 32, 213-219 (2009).

11. Ylikoski, J., Xing-Qun, L., Virkkala, J. \& Pirvola, U. Blockade of c-Jun N-terminal kinase pathway attenuates gentamicin-induced cochlear and vestibular hair cell death. Hear Res 166, 33-43 (2002).

12. Nakamagoe, M., Tabuchi, K., Uemaetomari, I., Nishimura, B. \& Hara, A. Estradiol protects the cochlea against gentamicin ototoxicity through inhibition of the JNK pathway. Hear Res 261, 67-74, doi:10.1016/j.heares.2010.01.004 (2010).

13. Wang, J. et al. A peptide inhibitor of c-Jun N-terminal kinase protects against both aminoglycoside and acoustic trauma-induced auditory hair cell death and hearing loss. J Neurosci 23, 8596-8607 (2003).

14. Sano, H. et al. Effect of geranylgeranylacetone on gentamycin ototoxicity in rat cochlea culture. Auris Nasus Larynx 34, 1-4, doi:10.1016/j.anl.2006.05.020 (2007).

15. Yu, Y., Szczepek, A. J., Haupt, H. \& Mazurek, B. Geldanamycin induces production of heat shock protein 70 and partially attenuates ototoxicity caused by gentamicin in the organ of Corti explants. J Biomed Sci 16, 79, doi:10.1186/14230127-16-79 (2009).

16. Yang, T. H., Young, Y. H. \& Liu, S. H. EGb 761 (Ginkgo biloba) protects cochlear hair cells against ototoxicity induced by gentamicin via reducing reactive oxygen species and nitric oxide-related apoptosis. J Nutr Biochem 22, 886-894, doi:10.1016/j.jnutbio.2010.08.009 (2011).

17. Kim, H. J. et al. Heme oxygenase-1 attenuates the cisplatin-induced apoptosis of auditory cells via down-regulation of reactive oxygen species generation. Free Radic Biol Med 40, 1810-1819, doi:10.1016/j.freeradbiomed.2006.01.018 (2006).

18. Floridi, A. et al. Effect of lonidamine on the energy metabolism of Ehrlich ascites tumor cells. Cancer Res 41, 4661-4666 (1981).

19. Cheng, C. Y. et al. Two new male contraceptives exert their effects by depleting germ cells prematurely from the testis. Biol Reprod 65, 449-461 (2001).

20. Shao, J. et al. Adjudin attenuates lipopolysaccharide (LPS)- and ischemia-induced microglial activation. J Neuroimmunol 254, 83-90, doi:10.1016/ j.jneuroim.2012.09.012 (2013).

21. Choung, Y. H. et al. Generation of highly-reactive oxygen species is closely related to hair cell damage in rat organ of Corti treated with gentamicin. Neuroscience 161, 214-226, doi:10.1016/j.neuroscience.2009.02.085 (2009).

22. Setsukinai, K., Urano, Y., Kakinuma, K., Majima, H. J. \& Nagano, T. Development of novel fluorescence probes that can reliably detect reactive oxygen species and distinguish specific species. J Biol Chem 278, 3170-3175, doi:10.1074/ jbc.M209264200 (2003).

23. Fan, J., Cai, H. \& Tan, W. S. Role of the plasma membrane ROS-generating NADPH oxidase in CD34+ progenitor cells preservation by hypoxia. J Biotechnol 130, 455-462, doi:10.1016/j.jbiotec.2007.05.023 (2007).

24. Salt, A. N., Kellner, C. \& Hale, S. Contamination of perilymph sampled from the basal cochlear turn with cerebrospinal fluid. Hear Res 182, 24-33 (2003).

25. Zheng, J. L. \& Gao, W. Q. Differential damage to auditory neurons and hair cells by ototoxins and neuroprotection by specific neurotrophins in rat cochlear organotypic cultures. Eur J Neurosci 8, 1897-1905 (1996).

26. Someya, S. et al. Sirt 3 mediates reduction of oxidative damage and prevention of age-related hearing loss under caloric restriction. Cell 143, 802-812, doi:10.1016/ j.cell.2010.10.002 (2010).

27. Kim, H. S. et al. SIRT3 is a mitochondria-localized tumor suppressor required for maintenance of mitochondrial integrity and metabolism during stress. Cancer Cell 17, 41-52, doi:10.1016/j.ccr.2009.11.023 (2010).

28. Hirschey, M. D. et al. SIRT3 regulates mitochondrial fatty-acid oxidation by reversible enzyme deacetylation. Nature 464, 121-125, doi:10.1038/nature08778 (2010).

29. Houtkooper, R. H., Pirinen, E. \& Auwerx, J. Sirtuins as regulators of metabolism and healthspan. Nat Rev Mol Cell Biol 13, 225-238, doi:10.1038/nrm3293 (2012).

30. Li, Y., Ding, D., Jiang, H., Fu, Y. \& Salvi, R. Co-administration of cisplatin and furosemide causes rapid and massive loss of cochlear hair cells in mice. Neurotox Res 20, 307-319, doi:10.1007/s12640-011-9244-0 (2011).

31. Schacht, J., Talaska, A. E. \& Rybak, L. P. Cisplatin and aminoglycoside antibiotics: hearing loss and its prevention. Anat Rec(Hoboken) 295, 1837-1850, doi:10.1002/ ar.22578 (2012)

32. Lee, J. H. et al. Different uptake of gentamicin through TRPV1 and TRPV4 channels determines cochlear hair cell vulnerability. Exp Mol Med 45, e12, doi:10.1038/emm.2013.25 (2013).

33. McFadden, S. L., Ding, D., Jiang, H. \& Salvi, R. J. Time course of efferent fiber and spiral ganglion cell degeneration following complete hair cell loss in the chinchilla. Brain Res 997, 40-51 (2004).

\section{Acknowledgments}

This study is supported by funds from the Chinese Ministry of Science and Technology (2013CB945604 to W.X. and W.Q.G.; 2012 CB966800 to W.Q.G.; 2011 CB504503 to S.Y.; and 2012CB967903), the National Natural Science Foundation of China (31270032 to W.X., 81130038 and 81372189 to W.Q.G.,30925035 to S.Y., 81300823 to L.X.), Science and 
Technology Commission of Shanghai Municipality (Pujiang program to W.Q.G.), Shanghai Education Committee Key Discipline and Specialty Foundation (J50208 to W.Q.G.), Shanghai Health Bureau Key Discipline and Specialty Foundation (to W.Q.G.), SJTU KC Wong foundation (to W.Q.G.).

\section{Author contributions}

W.X., W.-Q.G. and Y.Q. designed research; Y.Q., L.X. and J.S. performed research; W.X., W.-Q.G., S.Y. and C.Y.C. contributed new reagents/analytic tools; Y.Q., L.X., J.S., C.Y.C., W.X. and W.-Q.G. analyzed data; and Y.Q., L.X., W.-Q.G. and W.X. wrote the paper. L.X. performed the animal experiments; Y.Q. prepared figures 1,2,4,5; J.S. prepared figure 3; L.X. prepared figures 6,7. All authors reviewed the manuscript.

\section{Additional information}

Supplementary information accompanies this paper at http://www.nature.com/ scientificreports
Competing financial interests: The authors declare no competing financial interests.

How to cite this article: Quan, Y. et al. Adjudin protects rodent cochlear hair cells against gentamicin ototoxicity via the SIRT3-ROS pathway. Sci. Rep. 5, 8181; DOI:10.1038/ srep08181 (2015)

(c) (1) (2) This work is licensed under a Creative Commons Attribution-NonCommercialShareAlike 4.0 International License. The images or other third party material in this article are included in the article's Creative Commons license, unless indicated otherwise in the credit line; if the material is not included under the Creative Commons license, users will need to obtain permission from the license holder in order to reproduce the material. To view a copy of this license, visit http:// creativecommons.org/licenses/by-nc-sa/4.0/ 